\title{
Tailored long-term immunosuppressive regimen for adult liver transplant recipients with hepatocellular carcinoma
}

\author{
Sung-Hwa Kang, Shin Hwang, Tae-Yong Ha, Gi-Won Song, Dong-Hwan Jung, Ki-Hun Kim, \\ Chul-Soo Ahn, Deok-Bog Moon, Gil-Chun Park, Bo-Hyun Jung, Young-In Yoon, and Sung-Gyu Lee
Division of Hepatobiliary Surgery and Liver Transplantation, Department of Surgery, Asan Medical Center, University of Ulsan College of Medicine, Seoul, Korea

\begin{abstract}
Backgrounds/Aims: There are few guidelines for tailored immunosuppressive regimens for liver transplantation (LT) recipients with hepatocellular carcinoma $(\mathrm{HCC})$. To establish long-term immunosuppressive regimens suitable for Korean adult LT recipients, we analyzed those that were currently in use at a single high-volume institution. Methods: This cross-sectional study comprises three parts including review of the immunosuppressive regimens used to manage 2,147 adult LT outpatients, review of LT recipients who were diagnosed of HCC at LT, and review of LT recipients who suffered from HCC recurrence. Results: In 1,000 adult LT recipients who were living more than 5 years with no adverse events, 916 received a calcineurin inhibitor (CNI)-based therapy (CNI only in 520; $\mathrm{CNI}$ with mycophenolate mofetil [MMF] in 396) and 84 were receiving an MMF-based therapy (MMF only in 45; MMF with minimal CNI in 39). Tacrolimus was preferred over cyclosporine for both monotherapy and combination therapy along the passage of posttransplant period. There was no difference in selection of immunosuppressants, target blood concentration, and rate of combination therapy between LT recipients with and without HCC, except for the first 1 year. Sirolimus-based regimens were applied in 21 patients who showed HCC recurrence. Sorafenib was often used after conversion to sirolimus. Conclusions: Tailored immunosuppressive regimen covering the long-term posttransplant period should be established after consideration of individualized patient profiles including HCC. (Korean J Hepatobiliary Pancreat Surg 2014;18:48-51)
\end{abstract}

Key Words: Immunosuppressive regimen; Liver transplantation; Hepatocellular carcinoma; Calcineurin inhibitor; Sirolimus

\section{INTRODUCTION}

Liver transplantation (LT) has been an established treatment for end-stage liver diseases for more than 20 years, but there are currently few institutional protocols on long-term immunosuppression. Furthermore, there are few practical guidelines for tailored or optimized immunosuppressive regimens for LT recipients with hepatocellular carcinoma (HCC). Especially in Korea, HCC is concurrently diagnosed in a half of explant livers. It is thus necessary to establish long-term immunosuppressive therapy protocols that will achieve long-term stability in adult Korean LT recipients. ${ }^{1-3}$ Having performed $>3,000$ LT operations over the last 15 years, we feel that there is a real practical need to establish tailored long-term immunosuppressive regimens that are applicable in practice.
To optimize such regimens, we analyzed those that are currently in use at our high-volume single institution.

\section{MATERIALS AND METHODS}

This study comprises three parts: 1) a cross-sectional review of the immunosuppressive regimens used to manage all adult LT outpatients; 2) a cross-sectional study of $\mathrm{LT}$ recipients who were diagnosed of $\mathrm{HCC}$ at LT; and 3) a cross-sectional study of LT recipients who suffered from HCC recurrence.

Initially, we reviewed the immunosuppression status of 2,147 patients who visited our outpatient clinic regularly during the 15 months from October 2010 to December 2011. Of these cases, 1,000 adult LT patients who survived for more than 5 years posttransplant were also se-

Received: February 15, 2014; Revised: May 15, 2014; Accepted: May 18, 2014

Corresponding author: Shin Hwang

Division of Hepatobiliary Surgery and Liver Transplantation, Department of Surgery, Asan Medical Center, University of Ulsan College of Medicine, 388-1 Poongnap-dong, Songpa-gu, Seoul 138-736, Korea

Tel: +82-2-3010-3930, Fax: +82-2-3010-6701, E-mail: shwang@amc.seoul.kr

This study was partially supported by the Organ Transplantation Center of Asan Medical Center and Astellas Pharma Korea (2012-0825). 
lected to make long-term reference data on immunosuppressant therapeutic drug monitoring (TDM). TDM for tacrolimus, cyclosporine, mycophenolate mofetil (MMF), and sirolimus was routinely performed at every visit to the outpatient clinic. Sirolimus was primarily used to treat patients who suffered from HCC recurrence.

The target trough levels of tacrolimus with and without mycophenolate are summarized at Table 1. The suggested target levels of tacrolimus for HCC patients beyond the Milan criteria are quite comparable to the levels of CNI-sparing tacrolimus. Patient medical records were primarily reviewed retrospectively and partially prospectively. Our study protocol was approved by the institutional review board of our institution.

\section{RESULTS}

Initial cross-sectional study revealed that the immunosuppressive regimens used to manage 2,147 adult LT recipients could be classified into two types: calcineurin inhibitor (CNI)-based $(n=1,928,98.8 \%)$ and MMF-based

Table 1. The target trough levels of tacrolimus with and without mycophenolate mofetil (MMF) relative to the posttransplant period after adult living-donor liver transplantation. The values of calcineurin inhibitor (CNI)-sparing tacrolimus are comparable to the suggested CNI target levels for hepatocellular carcinoma patients beyond the Milan criteria

\begin{tabular}{ccc}
\hline \multirow{2}{*}{$\begin{array}{c}\text { Posttransplant } \\
\text { period }\end{array}$} & \multicolumn{2}{c}{ Target tacrolimus level (ng/ml) } \\
\cline { 2 - 3 } & $\begin{array}{c}\text { Tacrolimus } \\
\text { monotherapy }\end{array}$ & $\begin{array}{c}\text { CNI-sparing with } \\
\text { MMF }\end{array}$ \\
\hline 1 mo & $10-15$ & $7-10$ \\
$2-3$ mos & $10-12$ & $5-8$ \\
$3-12$ mos & $8-10$ & $5-8$ \\
$2-3$ yrs & $6-8$ & $<5$ \\
$3-5$ yrs & $5-7$ & $<5$ \\
$>5$ yrs & $\leq 5$ & $<5$ \\
\hline
\end{tabular}

$(\mathrm{n}=219,10.3 \%)$. CNI-based regimens included CNI monotherapy $(\mathrm{n}=1,021,47.6 \%$; tacrolimus in 765 [35.6\%] and cyclosporine in 256 [11.9\%] LT recipients), tacrolimus with low-dose MMF ( $\mathrm{n}=759,35.4 \%)$, and cyclosporine with low-dose MMF ( $n=148,6.9 \%)$. MMF-based regimens included MMF monotherapy ( $\mathrm{n}=107,5.0 \%)$, MMF with minimal tacrolimus $(\mathrm{n}=83,3.9 \%)$, and MMF with minimal cyclosporine $(\mathrm{n}=29,1.49 \%)$. Another 21 patients received sirolimus-based regimens, and all of these patients had HCC recurrence.

The cross-sectional study for 1,000 selected adult LT recipients who were followed for more than 5 years revealed that immunosuppressive regimens were classified as CNI-based $(n=916,91.6 \%)$ and MMF-based $(n=84$, $8.4 \%$ ) regimens. CNI-based regimens included CNI monotherapy ( $\mathrm{n}=520,52.0 \%$; tacrolimus in 411 [41.16\%] and cyclosporine in 109 [10.9\%]), tacrolimus with low-dose MMF ( $\mathrm{n}=354,35.4 \%$ ), and cyclosporine with low-dose MMF ( $\mathrm{n}=32,3.2 \%)$. MMF-based regimens included MMF monotherapy $(\mathrm{n}=45,4.5 \%)$, MMF with minimal tacrolimus ( $\mathrm{n}=32,3.2 \%)$, and MMF with minimal cyclosporine $(n=7,0.7 \%)$. The incidences of use of these therapies show that tacrolimus is preferred over cyclosporine for both monotherapy and combination therapy. Roughly a half of our patients received MMF for CNI-sparing therapy or monotherapy. Detailed long-term TDM data on immunosuppressant blood concentration are summarized at Table 2.

Among the $62 \mathrm{LT}$ patients who survived for more than 10 years posttransplant, the immunosuppressive regimens consisted of CNI monotherapy in 50 patients, CNI-MMF combination therapy in 9 patients, and MMF monotherapy in 3 patients. The target tacrolimus trough level at 10 years was intentionally adjusted to be $2 \mathrm{ng} / \mathrm{ml}$. The target mycophenolic acid trough level during MMF monotherapy was set to be $2 \mu \mathrm{g} / \mathrm{ml}$ for patients who survived

Table 2. Mean 12-hour trough levels of tacrolimus (Tac) and mycophenolate mofetil (MMF) in adult liver transplant outpatients relative to the posttransplant period

\begin{tabular}{|c|c|c|c|c|c|c|}
\hline \multirow{2}{*}{$\begin{array}{l}\text { Posttransplant } \\
\text { period }\end{array}$} & \multirow{2}{*}{$\begin{array}{c}\text { Tac mono } \\
\text { Tac (ng/ml) }\end{array}$} & \multicolumn{2}{|c|}{$\mathrm{Tac}+\mathrm{MMF}$} & \multicolumn{2}{|c|}{$\mathrm{Tac}+$ minimal $\mathrm{MMF}$} & \multirow{2}{*}{$\begin{array}{c}\text { MMF mono } \\
\text { MPA }(\mu \mathrm{g} / \mathrm{ml})\end{array}$} \\
\hline & & Tac (ng/ml) & MPA $(\mu \mathrm{g} / \mathrm{ml})$ & Tac (ng/ml) & $\mathrm{MPA}(\mu \mathrm{g} / \mathrm{ml})$ & \\
\hline $5-7$ years & $4.2 \pm 1.7$ & $3.3 \pm 2.3$ & $1.2 \pm 0.9$ & $<1.2$ & $2.1 \pm 1.1$ & $2.3 \pm 0.9$ \\
\hline $8-10$ years & $2.5 \pm 2.1$ & $2.2 \pm 1.6$ & $1.2 \pm 0.6$ & $<1.2$ & $1.6 \pm 1.4$ & $2.4 \pm 1.2$ \\
\hline$>10$ years & $2.2 \pm 0.4$ & $2.1 \pm 0.8$ & $1.4 \pm 0.2$ & $<1.2$ & $1.5 \pm 1.0$ & $1.9 \pm 0.8$ \\
\hline
\end{tabular}

MPA, mycophenolic acid. 
for more than 5 years.

There was no difference in selection of immunosuppressive agents, target blood concentration, and rate of combination therapy between LT recipients with and without HCC at the explant livers, except for the first 1 year. There was a trend on reduced trough level of tacrolimus during the first year in HCC recipients especially beyond the Milan criteria, but no statistical difference was identified after crude comparison of the mean values.

During the study period, 10 patients with HCC recurrence were prospectively followed up in order to reveal the usage patterns of sirolimus. The target sirolimus level as sirolimus monotherapy was set to be $10 \mathrm{ng} / \mathrm{ml}$ at posttransplant 1 year and $5 \mathrm{ng} / \mathrm{ml}$ at 5 years. Within 1 month after detection of HCC recurrence, precedent CNI usage was converted to sirolimus with or without low-dose CNI. Of them, 2 recipients were dropped out from sirolimus switching due to intractable side-effects. At first, high-dose sirolimus $>10 \mathrm{ng} / \mathrm{ml}$ was attempted with anticipation of anti-tumor effect, but a half of patients could not tolerate such high concentration, thus being no longer attempted. Combination of sirolimus and sorafenib was successfully installed in 6 of 8 patients within 1 month after conversion to sirolimus monotherapy. Three of these 6 patients showed rapid progression of HCC leading to death, but other 3 patients showed at least no definite progression of HCC for more than 6 months. One of these 6 patients showed clinical acute rejection, thus sirolimus monotherapy was changed to sirolimus with reduced-dose tacrolimus.

\section{DISCUSSION}

This cross-sectional study shows that the use of long-term immunosuppressive regimens in adult LT recipients were slowly and carefully adjusted after considering the overall posttransplant clinical courses of each patient (e.g., episodes of late acute rejection, infection, graft dysfunction, renal function). ${ }^{4}$ Our policy for long-term immunosuppression is to maintain immunosuppressant levels within a certain target range because immunosuppression at subtherapeutic levels for prolonged periods can induce subclinical graft damage and late graft dysfunction. ${ }^{5-7}$ In fact, the withdrawal of immunosuppressive agents has been attempted in a few studies, most especially in pedia- tric patients, but their final outcomes appear to be unpromising and possibly even harmful. ${ }^{8,9}$ It is currently inevitable therefore that posttransplant period-adjusted immunosuppression will need to be maintained for life. ${ }^{10,11}$

The long-term side effects of various immunosuppressive agents need to be considered also. Because CNI is often associated with nephrotoxicity and metabolic syndromes, renal-sparing treatment is often possible with CNI-MMF combination therapy. In contrast, the target level of CNI is much lower after 5 years than it is in earlier periods, thus CNI-sparing effects are often diluted. ${ }^{4}$ We currently suggest avoiding the use of combination immunosuppressive therapy after 10 years in order to prevent excessive immunosuppression. Recently, the role of a late liver biopsy after 10 years in LT recipients has become more important than before because various subclinical liver abnormalities have been reported.,

In this study, the most common immunosuppressive agent administered to adult LT recipients was tacrolimus. Recently, the availability of $0.25 \mathrm{mg}$ generic tacrolimus enabled us to meticulously adjust the trough level. The second-most common agent was MMF, but this agent has a wide range of bioavailability. We previously reported that the interindividual variability in MPA concentration is as wide as that of tacrolimus. ${ }^{4}$ We emphasize the need for MPA TDM in order to provide reliable dosage adjustment and prevent under- or over-immunosuppression.

It is reasonable to reduce the target trough level of tacrolimus during the first year in HCC recipients because high CNI concentration is known to be a significant risk factor for HCC recurrence. ${ }^{12}$ It was reported that the risk factors significantly and independently associated with HCC recurrence were exposure to higher levels of immunosuppression, preoperative serum alpha-fetoprotein $>50 \mathrm{ng} / \mathrm{ml}$, poorly differentiated histologic grading, and presence of microvascular invasion. ${ }^{13}$ Special attention should be paid to the patients with above-mentioned risk factors of HCC recurrence as well the HCC patients beyond the Milan criteria.

In contrast, we did not find any statistical difference in immunosuppressant trough levels after first year regardless of presence of explant HCC. This trend is probably based on the timing of $\mathrm{HCC}$ recurrence, in which about two thirds of HCC recurrences occur within the first year and most of them also happen within the first 3 years. ${ }^{14}$ 
Nowadays, sirolimus is more frequently used worldwide than before, especially to treat patients with HCC recurrence. The use of sirolimus-based immunosuppression regimens have been reported to reduce posttransplant HCC recurrence rates, although this is still a matter for debate. ${ }^{15,16}$ Furthermore, the effect of conversion to sirolimus after HCC recurrence was not demonstrated yet. ${ }^{17,18}$ For patients with HCC recurrence, sorafenib administration has been attempted after conversion to sirolimus. ${ }^{19-22}$ Sirolimus does not induce nephrotoxicity, thus sirolimus with or without reduced-dose MMF seems to be beneficial for patients who are unsuitable for MMF monotherapy due to MMF-associated side effects. ${ }^{4,23,24}$

In conclusion, HCC became a common indication of LT, especially in Asian countries where living-donor LT is frequently performed. Tailored immunosuppressive regimens covering the long-term posttransplant period should be established after consideration of individualized patient profiles including $\mathrm{HCC}$.

\section{REFERENCES}

1. Lee SG, Hwang S, Kim KH, Ahn CS, Moon DB, Ha TY, et al. Toward 300 liver transplants a year. Surg Today 2009; 39:367-373.

2. Hwang S, Lee SG, Ahn CS, Kim KH, Moon DB, Ha TY, et al. An increase in deceased donor incidence alleviated the need for urgent adult living donor liver transplantation in a Korean high-volume center. Transplant Proc 2010;42:1497-1501.

3. Lee SG, Moon DB, Shin H, Kim KH, Ahn CS, Ha TY, et al. Living donor liver transplantation for hepatocellular carcinoma: current status in Korea. Transplant Proc 2012;44:520-522.

4. Hwang S, Lee SG, Ahn CS, Kim KH, Moon DB, Ha TY, et al. A clinical assessment of mycophenolate drug monitoring after liver transplantation. Clin Transplant 2010;24:E35-E42.

5. Ekong UD. The long-term liver graft and protocol biopsy: do we want to look? What will we find? Curr Opin Organ Transplant 2011;16:505-508.

6. Hübscher SG. What is the long-term outcome of the liver allograft? J Hepatol 2011;55:702-717.

7. Mells G, Mann C, Hubscher S, Neuberger J. Late protocol liver biopsies in the liver allograft: a neglected investigation? Liver Transpl 2009;15:931-938.

8. Feng S, Ekong UD, Lobritto SJ, Demetris AJ, Roberts JP, Rosenthal P, et al. Complete immunosuppression withdrawal and subsequent allograft function among pediatric recipients of parental living donor liver transplants. JAMA 2012;307:283-293.

9. Sánchez-Fueyo A. Hot-topic debate on tolerance: immunosuppression withdrawal. Liver Transpl 2011;17 Suppl 3:S69-S73.
10. Porrett P, Shaked A. The failure of immunosuppression withdrawal: patient benefit is not detectable, inducible, or reproducible. Liver Transpl 2011;17 Suppl 3:S66-S68.

11. Jain A, Sharma R, Ryan C, Tsoulfas G, Orloff M, Abt P, et al. Mycophenolate mofetil in combination with tacrolimus in adult living donor liver transplant recipients. J Korean Soc Transplant 2006;20:84-89.

12. Vivarelli M, Cucchetti A, Piscaglia F, La Barba G, Bolondi L, Cavallari A, et al. Analysis of risk factors for tumor recurrence after liver transplantation for hepatocellular carcinoma: key role of immunosuppression. Liver Transpl 2005;11:497-503.

13. Vivarelli M, Cucchetti A, La Barba G, Ravaioli M, Del Gaudio M, Lauro A, et al. Liver transplantation for hepatocellular carcinoma under calcineurin inhibitors: reassessment of risk factors for tumor recurrence. Ann Surg 2008;248:857-862.

14. Hwang S, Moon DB, Ahn CS, Kim KH, Ha TY, Song GW, et al. Risk-based long-term screening for hepatocellular carcinoma recurrence after living donor liver transplantation. Transplant Proc 2013;45:3076-3084.

15. Vivarelli M, Dazzi A, Cucchetti A, Gasbarrini A, Zanello M, Di Gioia P, et al. Sirolimus in liver transplant recipients: a large single-center experience. Transplant Proc 2010;42:2579-2584.

16. Toso C, Merani S, Bigam DL, Shapiro AM, Kneteman NM. Sirolimus-based immunosuppression is associated with increased survival after liver transplantation for hepatocellular carcinoma. Hepatology 2010;51:1237-1243.

17. Kornberg A, Küpper B, Tannapfel A, Thrum K, Wilberg J, Bärthel E, et al. Adjuvant conversion to sirolimus in liver transplant patients with recurrent hepatocellular carcinoma - preliminary results. Transpl Int 2008;21:96-99.

18. Kornberg A, Küpper B, Tannapfel A, Katenkamp K, Thrum K, Habrecht $\mathrm{O}$, et al. Long-term survival after recurrent hepatocellular carcinoma in liver transplant patients: clinical patterns and outcome variables. Eur J Surg Oncol 2010;36:275-280.

19. Gomez-Martin C, Bustamante J, Castroagudin JF, Salcedo M, Garralda E, Testillano M, et al. Efficacy and safety of sorafenib in combination with mammalian target of rapamycin inhibitors for recurrent hepatocellular carcinoma after liver transplantation. Liver Transpl 2012;18:45-52.

20. Bhoori S, Toffanin S, Sposito C, Germini A, Pellegrinelli A, Lampis A, et al. Personalized molecular targeted therapy in advanced, recurrent hepatocellular carcinoma after liver transplantation: a proof of principle. J Hepatol 2010;52:771-775.

21. Waidmann O, Hofmann WP, Zeuzem S, Trojan J. mTOR inhibitors and sorafenib for recurrent heptocellular carcinoma after orthotopic liver transplantation. J Hepatol 2011;54:396-398.

22. Yoon DH, Ryoo BY, Ryu MH, Lee SG, Hwang S, Suh DJ, et al. Sorafenib for recurrent hepatocellular carcinoma after liver transplantation. Jpn J Clin Oncol 2010;40:768-773.

23. Masetti M, Montalti R, Rompianesi G, Codeluppi M, Gerring R, Romano A, et al. Early withdrawal of calcineurin inhibitors and everolimus monotherapy in de novo liver transplant recipients preserves renal function. Am J Transplant 2010;10:2252-2262.

24. Perrakis A, Schwarz K, Yedibela S, Croner RS, Hohenberger W, Müller V. Impact of the conversion of the immunosuppressive regimen from prograf to advagraf or to sirolimus in long-term stable liver transplant recipients: indications, safety, and outcome. Transplant Proc 2011;43:3702-3707. 